\title{
GW23-e0832 IMPROVE THE TRANSVENOUS EXTRACTION OF CARDIAC IMPLANTABLE ELECTRONIC DEVICES LEAD SUCCESS BY THE ABLATION CATHETER FROM FEMORAL APPROACH
}

doi:10.1136/heartjnl-2012-302920m.2

Xian-Hui Zhou, Jin-Xin Li, Yao-dong Li, Yu Zhang, Yan-Yi Zhang, Bao-peng Tang. First Affiliated Hospital of XinJiang Medical University

Objectives Complete extraction the cardiac implantable electronic devices (CIED) lead systems remains a complex procedure. This retrospective study was conducted to describe the safety and effectiveness of a modified extracting transvenous lead technique.

Methods Total 151 patients, 97 males and 54 females, with CIED related infections were retrospective enrolled in this study. There were 75 patients with CIED lead extraction using standard techniques and equipment, the other 76 patients with CIED lead extraction using a modified extraction transvenous lead techniques.

Results There were no significant differences in the age, gender, NYHA functional class in two groups. The lead extraction time, procedure time and fluoroscopy exposure time shows significant reduction (lead extraction time from $33 \pm 8 \mathrm{~min}$ to $22 \pm 7 \mathrm{~min}$, $\mathrm{p}<0.001$; procedure time from $64 \pm 18 \mathrm{~min}$ to $47 \pm 19 \mathrm{~min}, \mathrm{p}<0.001$ and fluorocopy exposure time from $36 \pm 7 \mathrm{~min}$ to $24 \pm 6 \mathrm{~min}$. $\mathrm{p}<0.001)$. There was significant improvement in Cumulative successive rate of lead extraction from 69 of 75 (92\%) patients by using the standard technique and equipments, to 73 of 76 (96.1\%) patients by the modified extraction of transvenous lead techniques. $(p=0.03)$.

Conclusions It is effective and safe extraction CIED lead through femoral approach by the modified extraction of transvenous lead techniques for ablation catheter. 